\title{
Effects of emphysema on oxygen uptake during maximal exercise in COPD
}

\author{
Massimo Miniati - Giosuè Angelo Catapano • \\ Simonetta Monti - Francesca Mannucci • \\ Matteo Bottai
}

Received: 2 December 2010/ Accepted: 17 March 2011

(C) SIMI 2011

\begin{abstract}
The objective of the study is to assess the effects of emphysema on peak oxygen uptake $\left(\dot{V} \mathrm{O}_{2 \text { peak }}\right)$ during a cardiopulmonary exercise test in patients with chronic obstructive pulmonary disease (COPD). We measured $\dot{V} \mathrm{O}_{2 \text { peak }}$ and oxygen pulse in 80 patients with stable COPD exercising maximally. Oxygen saturation was measured by pulse oximetry $\left(\mathrm{SpO}_{2}\right)$, and the ventilatory response assessed by the ratio of tidal volume $\left(V_{\mathrm{T}}\right)$ at peak to slow vital capacity (SVC) at baseline, and by the percent increase of peak $V_{\mathrm{T}}$ over baseline. Computed tomography imaging (CT scan) served as the reference diagnostic standard for emphysema. Based on the panel-grading (PG) method, emphysema was rated absent or mild $(\mathrm{PG} \leq 30$, $n=54)$, or moderate to severe (PG $>30, n=26)$.
\end{abstract}

Electronic supplementary material The online version of this article (doi:10.1007/s11739-011-0575-x) contains supplementary material, which is available to authorized users.

\section{Miniati $(\square)$}

Department of Medical and Surgical Critical Care,

University of Florence, Viale Morgagni 85,

50134 Florence, Italy

e-mail: Massimo.Miniati@unifi.it

G. A. Catapano - S. Monti · F. Mannucci

Tuscany Foundation "Gabriele Monasterio", Pisa, Italy

S. Monti

Institute of Clinical Physiology, National Research Council,

Pisa, Italy

\section{Bottai}

Unit of Biostatistics, Department of Environmental Medicine,

Karolinska Institutet, Stockholm, Sweden

M. Bottai

Division of Biostatistics, Arnold School of Public Health, University of South Carolina, Columbia, SC, USA
Multiple quantile regression was applied to estimate the effects of $\mathrm{PG}>30$ on $\dot{V} \mathrm{O}_{2 \text { peak }}$. At peak exercise, the patients with $\mathrm{PG}>30$ had significantly lower $\dot{V} \mathrm{O}_{2}$, oxygen pulse and $\mathrm{SpO}_{2}$, and featured a blunted ventilatory response with respect to those with $\mathrm{PG} \leq 30(p<0.001)$. With multiple quantile regression, the effects of $\mathrm{PG}>30$ on $\dot{V} \mathrm{O}_{2 \text { Peak }}$ were only partially explained by the degree of lung hyperinflation, a substantial component being imputable to impairment of lung diffusing capacity. In conclusion, chronic obstructive pulmonary disease patients with moderate to severe emphysema feature significantly lower exercise tolerance than those with no or mild emphysema. Our findings underscore the need of tailoring therapeutic interventions for COPD to the predominant clinical phenotype to improve exercise capacity.

Keywords COPD - Emphysema - Exercise tolerance . Oxygen uptake

\section{Introduction}

Chronic obstructive pulmonary disease (COPD) is characterized by airflow limitation that is not fully reversible [1]. Airflow obstruction results from the combination of two mechanisms: increased airway resistance due to narrowing of the small airways, and loss of lung elastic recoil due to emphysema.

Over the years, evidence has emerged that patients with COPD and predominant emphysema differ remarkably from those with predominant airway disease [2-6].

A recent study, comprising 458 patients with established COPD, shows that patients with radiologic evidence of emphysema feature significantly lower body mass index, greater airflow obstruction, and more severe impairment of 
lung diffusing capacity than those without emphysema [5]. In addition, emphysemic patients were found to have greater restriction of physical activity and worse quality of life than non-emphysemic ones [5]. The latter findings were based on the results of a self-administered respiratory questionnaire [7].

The present study was undertaken to assess the effects of emphysema on peak oxygen uptake during a cardiopulmonary exercise test in patients with stable COPD. Volumetric CT scan was used as the reference standard for evaluating the presence and severity of emphysema.

\section{Methods}

Sample

The study sample comprises 80 consecutive patients with a diagnosis of COPD based on a post-bronchodilatator ratio of forced expiratory volume in $1 \mathrm{~s}$ to forced vital capacity $\left(\mathrm{FEV}_{1} / \mathrm{FVC}\right)<0.70$. All the patients were evaluated at the outpatient clinic, Tuscany Foundation "G. Monasterio", Pisa (Italy), between January 2008 and 2009.

Exclusion criteria were history of asthma, acute exacerbation of COPD in the 2 months preceding the study, unstable angina, recent myocardial infarction or stroke, heart failure (left ventricle ejection fraction $<50 \%$ on echocardiography), respiratory failure, poorly controlled systemic arterial hypertension or cardiac dysrhythmia, peripheral arteriopathy, or mental impairment precluding cooperation.

In all the patients, we obtained pulmonary function tests at baseline, including slow and forced expiratory flow rates and volumes, intrathoracic gas volume by body plethysmography, single breath-diffusing capacity of the lung for carbon monoxide $\left(\mathrm{DL}_{\mathrm{CO}}\right)$, and partial pressure of oxygen $\left(\mathrm{PaO}_{2}\right)$ and carbon dioxide $\left(\mathrm{PaCO}_{2}\right)$ in arterial blood. Spirometry, lung volumes and $\mathrm{DL}_{\mathrm{CO}}$ measurements were performed according to ATS/ERS standards [8-10]. Blood gases were measured while the patients were breathing room air. Lung function tests were obtained on the same day as the cardiopulmonary exercise test.

At the time of the study, all the patients were receiving at least one of the following drugs: inhaled long-acting beta-2-agonists (LABA, $n=68$ ), inhaled long-acting muscarinic antagonists (LAMA, $n=43$ ), inhaled corticosteroids (ICS, $n=68$ ), or oral theophylline $(n=11)$. For further details, see the online data supplement. The therapy had been prescribed by the attending physicians, and was not discontinued prior to entering the study. None of the patients had undergone any pulmonary rehabilitation program, and none were on long-term oxygen therapy.
The protocol was approved by the institutional ethics committee, and an informed written consent was obtained from all the patients before entering the study.

\section{Cardiopulmonary exercise test}

The cardiopulmonary exercise test (CPET) was performed on an electrically braked cycle ergometer $\left(V \mathrm{O}_{2 \max }\right.$ Encore CPX, SensorMedics, CA) according to the ATS/ACCP recommendations [11]. The protocol included three stages: resting, unloaded pedaling, and exercise. The external work rate was incremented continuously using the ramp protocol (rate of increment $10 \mathrm{~W} / \mathrm{min}$ ). The patients were encouraged to cycle until exhaustion. During the test, the electrocardiogram was monitored by telemetry, and the arterial oxygen saturation measured by pulse oximetry $\left(\mathrm{SpO}_{2}\right)$.

The slope of the rate of change in oxygen uptake divided by the rate of change in external work $\left(\Delta \dot{V} \mathrm{O}_{2} / \Delta\right.$ work $)$ was taken as an index of the aerobic work efficiency. Peak oxygen uptake ( $\left.\dot{V} \mathrm{O}_{2 \text { peak }}\right)$ and "oxygen pulse", or the ratio of $\dot{V} \mathrm{O}_{2}$ to the heart rate at peak exercise, were expressed as percent of their predicted value using appropriate reference equations [12].

The ventilatory response was assessed by the ratio of $V_{\mathrm{T}}$ at peak exercise to slow vital capacity (SVC) at baseline, and by the percent increase of $V_{\mathrm{T}}$ over baseline, that is $\left[\left(V_{\mathrm{T}}\right.\right.$ at peak exercise $-V_{\mathrm{T}}$ at baseline) $/ V_{\mathrm{T}}$ at baseline $] \times 100$.

The modified Borg scale was used to rate the intensity of breathing discomfort and of leg discomfort at rest and at peak exercise [13]. The patients were asked to specify the reason(s) why they stopped exercising.

The test was interrupted if any of the following were recorded: signs of ischemia on the electrocardiogram, hypertension (systolic blood pressure $>220 \mathrm{mmHg}$ or diastolic blood pressure $>120 \mathrm{mmHg}$ ), second or third degree heart block, or severe desaturation $\left(\mathrm{SpO}_{2} \leq 80 \%\right)$.

\section{Computed tomography}

Volumetric CT scan of the thorax was obtained within 2 weeks of CPET. It was performed on a 64-detector row scanner (Toshiba Aquilion, Japan) with the patient breath holding at full inspiration for $10 \mathrm{~s}$. No contrast medium was infused. Acquisition setting was $120 \mathrm{kVp}$ with mAs modulated according to the patient's attenuation as assessed before scan acquisition (range 60-250 mAs). Slice thickness was set at $0.65 \mathrm{~mm}$. Scans were reconstructed in the axial, sagittal and coronal planes. Images were viewed using a window level of -600 Hounsfield Units (HU) and a width of 1,500 $\mathrm{HU}$, and were examined independently by a chest radiologist and a chest physician for the presence of areas of low attenuation and vascular disruption. Both 
raters were blinded to lung function and CPET data. Maximum intensity projection technique was used to evaluate vascular disruption, and minimum intensity projection to highlight focal areas of low attenuation in the lung parenchyma [14].

The severity of emphysema was scored on a non-parametric scale from 0 (no emphysema) to 100 using the panel-grading (PG) method of Thurlbeck et al. [15].

This consists of 16 inflation-fixed, paper-mounted, midsagittal whole lung sections that are arranged at intervals of 5 between 0 and 50, and at intervals of 10 between 60 and 100. A PG of 5 or less is consistent with trace emphysema, a PG of 10-30 indicates mild emphysema, a PG $>30$ to 50 moderate emphysema, and a PG $>50$ to 100 severe emphysema. In scoring emphysema on CT, the two raters used sagittal lung sections and gave them the PG of the standard most closely similar, or a PG between two standards.

\section{Statistical analysis}

For the categorical variables, differences between emphysemic and non-emphysemic patients were tested for by Fisher's exact test. For the continuous variables, Mood's median test was used, and the exact $p$ value reported. Continuous variables are reported in the text and tables as median and interquartile range (IQR).

The scatter plot of the PG of emphysema by the two independent raters was tested for departure from perfect agreement by fitting a simple linear regression model and testing the null hypothesis that the intercept is equal to zero and the slope is equal to one, jointly. The resulting $p$ value was 0.329 , thus indicating no statistically significant difference in scoring emphysema. Therefore, the PG scores by the two independent raters were averaged. Multiple quantile regression was applied to estimate the effect of emphysema on the 25 th, median, and 75 th percentile of $\dot{V} \mathrm{O}_{2 \text { peak }}$ during $\mathrm{CPET}$. The dependent variable $\dot{V} \mathrm{O}_{2 \text { peak }}$ was expressed as percentage predicted. The independent variables were emphysema (absent or mild, $\mathrm{PG} \leq 30$ and moderate to severe, $\mathrm{PG}>30$ ), gender, ratio of inspiratory capacity to total lung capacity (IC/TCL), and $\mathrm{DL}_{\mathrm{CO}}$ as percentage predicted. In the text and tables, we give the estimates, 95\% confidence intervals, and $p$ values for different sets of independent variables. With 5,000 bootstrap replicates, we estimated a $96.6 \%$ power to detect the observed difference in median $\dot{V} \mathrm{O}_{2 \text { peak }}$ between the COPD patients with moderate to severe emphysema and those with no or only mild emphysema. Two-tailed $p$ values of less than 0.05 were considered statistically significant throughout.

\section{Results}

Emphysema was diagnosed consistently by the two independent raters in $34(43 \%)$ of 80 patients. The two raters assigned the same PG in 62 cases of whom 46 were considered as not having emphysema $(\mathrm{PG}=0)$. Among the emphysemic patients, the median PG was 45 (IQR 35-60).

Eight patients had mild emphysema (PG between 5 and 30 ), either paraseptal or centrilobular, predominantly distributed in the upper lobes; they did not differ from the 46 patients without emphysema as regards age, gender, cumulative smoking history, or baseline lung function.

We therefore divided the study sample in two groups as follows: (a) no or mild emphysema ( $\mathrm{PG} \leq 30, n=54$ ), (b) moderate to severe emphysema ( $\mathrm{PG}>30, n=26$ ).

The patients with PG $>30$ had significantly lower BMI, $\mathrm{FEV}_{1}, \mathrm{IC} / \mathrm{TCL}$ ratio, and $\mathrm{DL}_{\mathrm{CO}}$ than those with $\mathrm{PG} \leq 30$ (Table 1). There was no significant difference across the two groups with regard to age, gender, pack-years of smoking, or resting arterial blood gases (Table 1).

Most $(88 \%)$ of the patients with $\mathrm{PG}>30$ stopped exercising because of severe breathing discomfort, either alone or in combination with leg discomfort (Table 2) and such limitation occurred in $63 \%$ of the patients with $\mathrm{PG} \leq 30$.

Figure 1 shows the frequency distribution of structural emphysema in the study sample as a function of the maximum work load attained. The proportion of patients with PG $>30$ was significantly higher in the lower tertile than in the mid or upper tertile of the maximum work load.

The crude difference in the median value of maximum work load between the patients with PG $>30$ and those with $\mathrm{PG} \leq 30$ was $-20 \mathrm{~W}$ (Table 2). After adjusting for age and gender, the difference was $-29.44 \mathrm{~W}$. Both differences were highly statistically significant $(p<0.001)$.

At peak exercise, the patients with $\mathrm{PG}>30$ had significantly lower $\dot{V} \mathrm{O}_{2}$, oxygen pulse, and $\mathrm{SpO}_{2}$ than those with $\mathrm{PG} \leq 30$; also, they featured a blunted ventilatory response as reflected by the lower $V_{\mathrm{T}}$ peak/SVC ratio, and the smaller percent increase of peak $V_{\mathrm{T}}$ over baseline (Table 2). There was no significant difference between the two groups with regard to aerobic work efficiency (Table 2).

Among the patients with $\mathrm{PG}>30, \dot{V} \mathrm{O}_{2 \text { peak }}$ was significantly correlated with $\mathrm{DL}_{\mathrm{CO}}$, IC/TLC ratio, $\mathrm{FEV}_{1}$ and, to a smaller extent, with BMI (Table 3). In the patients with $\mathrm{PG} \leq 30, \dot{V} \mathrm{O}_{2 \text { peak }}$ correlated significantly with $\mathrm{DL}_{\mathrm{CO}}$ and IC/TLC, but not with $\mathrm{FEV}_{1}$ or BMI (Table 3). In both groups, there was a highly significant linear correlation between $\dot{V} \mathrm{O}_{2 \text { peak }}$ and the ventilatory response at peak exercise (Table 3 ). 
Table 1 Baseline characteristics of the study sample

\begin{tabular}{|c|c|c|c|c|}
\hline Characteristic & $\begin{array}{l}\text { All patients } \\
(n=80)\end{array}$ & $\begin{array}{l}\text { No or mild } \\
\text { emphysema }(n=54)\end{array}$ & $\begin{array}{l}\text { Moderate to severe } \\
\text { emphysema }(n=26)\end{array}$ & $p^{*}$ \\
\hline Age (years) & $70(64-73)$ & $68(64-72)$ & $71(67-75)$ & 0.811 \\
\hline Male sex & $62(78)$ & $41(76)$ & $21(81)$ & 0.777 \\
\hline $\operatorname{BMI}\left(\mathrm{kg} / \mathrm{m}^{2}\right)$ & $27(24-31)$ & $28(26-31)$ & $24(22-26)$ & 0.003 \\
\hline Current smokers & $14(18)$ & $11(20)$ & $3(12)$ & 0.531 \\
\hline Pack-years of smoking & $41(30-52)$ & $40(27-50)$ & $45(37-53)$ & 0.322 \\
\hline $\mathrm{FEV}_{1}(\%$ predicted $)$ & $57(40-71)$ & $65(54-77)$ & $38(34-54)$ & $<0.001$ \\
\hline IC/TLC (\%) & $39(31-45)$ & $43(36-46)$ & $29(25-34)$ & $<0.001$ \\
\hline $\mathrm{DL}_{\mathrm{CO}}(\%$ predicted $)$ & $74(63-88)$ & $81(72-97)$ & $51(41-67)$ & $<0.001$ \\
\hline Resting $\mathrm{PaO}_{2}(\mathrm{mmHg})$ & $75(70-81)$ & $77(72-81)$ & $71(66-82)$ & 0.470 \\
\hline Resting $\mathrm{PaCO}_{2}(\mathrm{mmHg})$ & $38(36-40)$ & $38(35-40)$ & $39(37-41)$ & 0.054 \\
\hline
\end{tabular}

Data are reported as median (interquartile range), or number $(\%)$

$B M I$ body mass index, $F E V_{l}$ forced expiratory volume in one second, $I C$ inspiratory capacity, $T L C$ total lung capacity, $D L_{C O}$ diffusing capacity of the lung for carbon monoxide, $\mathrm{PaO}_{2}$ partial pressure of oxygen in arterial blood, $\mathrm{PaCO}_{2}$ partial pressure of carbon dioxide in arterial blood

* No or mild versus moderate to severe emphysema

Table 2 Cardiopulmonary exercise test in COPD patients

\begin{tabular}{|c|c|c|c|c|}
\hline Measured variable & $\begin{array}{l}\text { All patients } \\
(n=80)\end{array}$ & $\begin{array}{l}\text { No or mild emphysema } \\
(n=54)\end{array}$ & $\begin{array}{l}\text { Moderate to severe } \\
\text { emphysema }(n=26)\end{array}$ & $p^{*}$ \\
\hline Breathing discomfort (Borg scale) & $57(71)$ & $34(63)$ & $23(88)$ & 0.019 \\
\hline Maximum work load (W) & $70(60-90)$ & $80(70-108)$ & $60(46-70)$ & $<0.001$ \\
\hline$\Delta \dot{V} \mathrm{O}_{2} / \Delta$ work $(\mathrm{mL} / \mathrm{min} / \mathrm{W})$ & $12(11-14)$ & $12(11-14)$ & $12(11-14)$ & 0.346 \\
\hline Heart rate at peak (cycles/min) & $119(108-130)$ & $119(111-133)$ & $114(105-123)$ & 0.235 \\
\hline Respiratory rate at peak (cycles/min) & $30(26-33)$ & $30(27-33)$ & $31(26-35)$ & 0.814 \\
\hline$\dot{V} \mathrm{O}_{2 \text { peak }}(\mathrm{mL} / \mathrm{min} / \mathrm{kg})$ & $12(10-15)$ & $13(11-16)$ & $10(9-11)$ & $<0.001$ \\
\hline$\dot{V} \mathrm{O}_{2 \text { peak }}(\%$ predicted $)$ & $49(37-62)$ & $55(46-67)$ & $34(29-44)$ & $<0.001$ \\
\hline Oxygen pulse at peak ( $\%$ predicted) & $63(49-78)$ & $71(59-81)$ & $44(40-62)$ & $<0.001$ \\
\hline $\mathrm{SpO}_{2}$ at peak $(\%)$ & $95(92-96)$ & $96(94-97)$ & $91(89-94)$ & $<0.001$ \\
\hline$V_{\mathrm{T}}$ at peak/SVC $(\%)$ & $43(36-48)$ & $45(40-50)$ & $35(32-41)$ & $<0.001$ \\
\hline Increase of peak $V_{\mathrm{T}}$ over baseline $(\%)$ & $125(72-156)$ & $133(103-174)$ & 75 (56-91) & $<0.001$ \\
\hline
\end{tabular}

Data are reported as median (interquartile range), or number $(\%)$

$\Delta$ rate of change, $\dot{V} \mathrm{O}_{2 \text { peak }}$ oxygen uptake at peak exercise, oxygen pulse $\dot{V} \mathrm{O}_{2} /$ heart rate at peak exercise, $\mathrm{SpO}_{2}$ arterial oxygen saturation by pulse oximeter, $V_{\mathrm{T}}$ tidal volume, $S V C$ slow vital capacity at baseline, increase in $V_{\mathrm{T}}$ over baseline $=\left[\left(V_{\mathrm{T}}\right.\right.$ at peak $-V_{\mathrm{T}}$ at baseline $) / V_{\mathrm{T}}$ at baseline $] \times 100$

* No or mild versus moderate to severe emphysema

Figure 2 shows the effects of $\mathrm{PG}>30$ on the median value of $\dot{V} \mathrm{O}_{2 \text { peak }}$ as estimated with multiple quantile regression.

The crude effect is estimated to be -21 . This means that the median $\dot{V} \mathrm{O}_{2 \text { peak }}$ is smaller in the patients with $\mathrm{PG}>30$ than in the others by 21 percentage points $(p<0.001)$. After adjusting by gender, the difference is only slightly reduced. After further adjusting for IC/TLC ratio, the difference drops somewhat, but remains statistically significant $(p=0.002)$. This means that there is an effect of $\mathrm{PG}>30$ on median $\dot{V} \mathrm{O}_{2 \text { peak, }}$, which is independent of the degree of hyperinflation at rest as reflected by IC/TLC ratio. Finally, when we further adjust for $\mathrm{DL}_{\mathrm{CO}}$ as percentage predicted, the difference nearly vanishes, and is no longer statistically significant $(p=0.889)$.

The interpretation of the effects of $\mathrm{PG}>30$ on the other quartiles of $\dot{V} \mathrm{O}_{2 \text { peak }}$ is analogous. However, the effects are larger on the median than on the first quartile, and even larger on the third quartile (see the online data supplement). This is the consequence of the distribution of $\dot{V} \mathrm{O}_{2 \text { peak }}$ values that is more spread in the patients with $\mathrm{PG} \leq 30$ than in those with PG $>30$ (Fig. 3). 


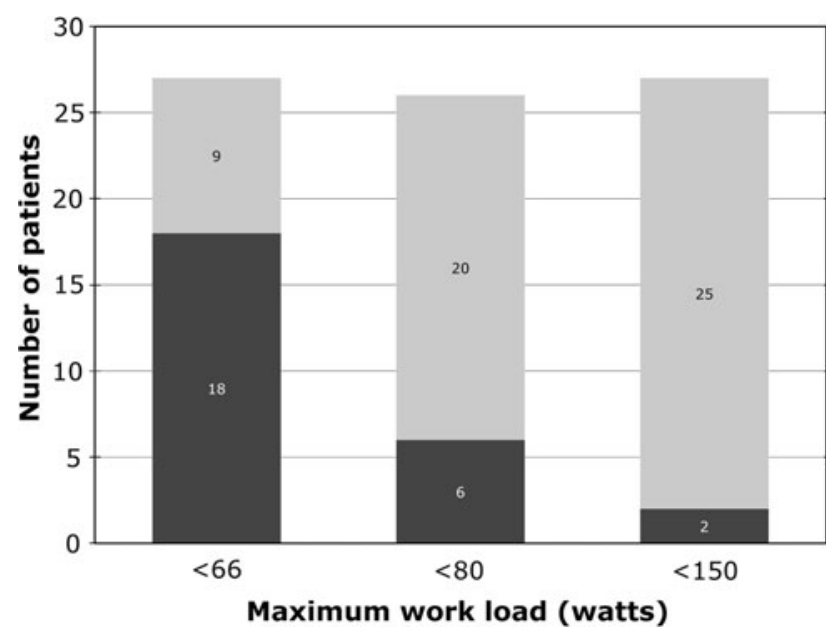

Fig. 1 Frequency distribution of structural emphysema as a function of the maximum work load (tertiles) in 80 patients with COPD. Black bars moderate to severe emphysema ( $\mathrm{PG}>30$ ). Gray bars no or only mild emphysema $(\mathrm{PG} \leq 30)$. The proportion of patients with PG $>30$ is significantly higher in the lower tertile than in the mid, or upper tertile ( $p=0.002$ and $p<0.001$, respectively)

\section{Discussion}

Our results can be summarized as follows: (a) patients with COPD and moderate to severe emphysema on CT have significantly greater impairment of baseline lung function than those with no, or only mild emphysema; (b) upon exercising maximally, emphysemic patients feature significantly lower $\dot{V} \mathrm{O}_{2 \text { peak }}$, oxygen pulse and $\mathrm{SpO}_{2}$, and exhibit a blunted ventilatory response with respect to nonemphysemic ones; (c) lung hyperinflation and impaired diffusing capacity contribute to exercise intolerance in patients with overt emphysema.

As to the mechanisms underlying the reduced exercise tolerance in COPD, Diaz et al. [16] find a significant linear

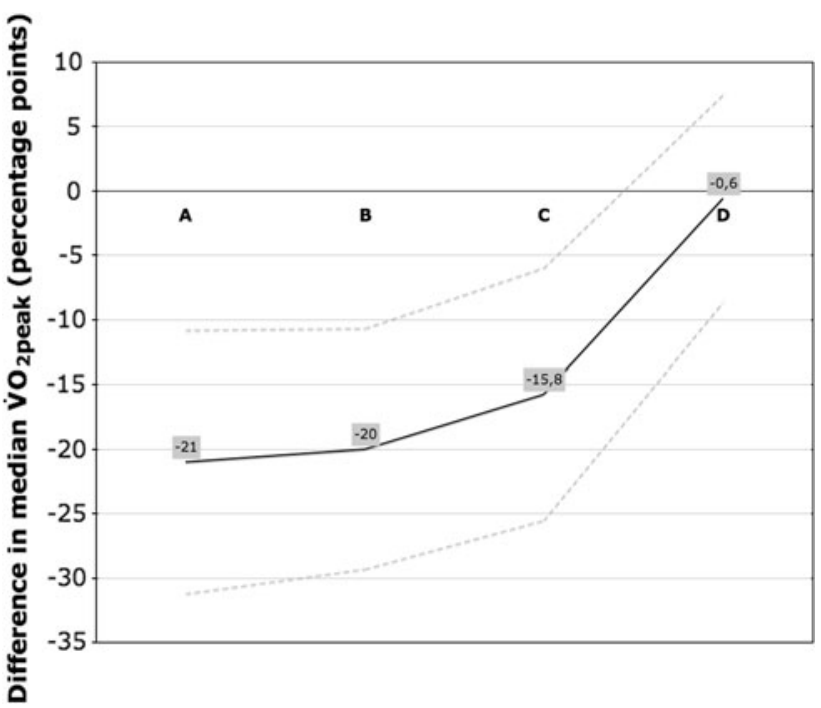

Fig. 2 Difference in median oxygen uptake at peak exercise $\left(\dot{V} \mathrm{O}_{2 \text { peak }}\right)$ between COPD patients with moderate to severe emphysema (PG > 30), and those with no or only mild emphysema $(\mathrm{PG} \leq 30)$. a Unadjusted difference $(p<0.001)$. b Adjusted for gender $(p<0.001)$. c Adjusted for gender, and IC/TLC ratio $(p=0.002)$. d Adjusted for gender, IC/TLC ratio, and $\mathrm{DL}_{\mathrm{CO}}$ as percentage predicted $(p=0.889)$. Dashed lines identify the $95 \%$ confidence interval of the difference in median $\dot{V} \mathrm{O}_{2 \text { peak }}$

correlation between baseline IC (as percentage predicted) and $\dot{V} \mathrm{O}_{2 \text { peak }}(r=0.750, p<0.001)$. In stepwise multiple regression, IC and $\mathrm{FEV}_{1} / \mathrm{FVC}$ ratio are the only significant contributors to the reduced exercise tolerance [16]. In that study, however, neither the lung diffusing capacity, nor the presence and severity of structural emphysema were assessed.

O'Donnell et al. [17] report that COPD patients fail to expand $V_{\mathrm{T}}$ in response to the increased metabolic demand imposed by maximal exercise. $\dot{V} \mathrm{O}_{2 \text { peak }}$ best correlates with

Table 3 Correlations with peak oxygen uptake during cardiopulmonary exercise test in COPD

\begin{tabular}{|c|c|c|c|c|c|c|}
\hline \multirow[t]{2}{*}{ Independent variable } & \multicolumn{2}{|c|}{ All patients $(n=80)$} & \multicolumn{2}{|c|}{ No or mild emphysema $(n=54)$} & \multicolumn{2}{|c|}{ Moderate to severe emphysema $(n=26)$} \\
\hline & $r$ & $p$ & $r$ & $p$ & $r$ & $p$ \\
\hline BMI $\left(\mathrm{kg} / \mathrm{m}^{2}\right)$ & 0.374 & $<0.001$ & 0.125 & 0.368 & 0.471 & 0.015 \\
\hline $\mathrm{FEV}_{1}(\%$ predicted $)$ & 0.403 & $<0.001$ & 0.058 & 0.677 & 0.653 & $<0.001$ \\
\hline IC/TLC (\%) & 0.608 & $<0.001$ & 0.366 & 0.006 & 0.728 & $<0.001$ \\
\hline $\mathrm{DL}_{\mathrm{CO}}(\%$ predicted $)$ & 0.642 & $<0.001$ & 0.516 & $<0.001$ & 0.628 & $<0.001$ \\
\hline Resting $\mathrm{PaO}_{2}(\mathrm{mmHg})$ & 0.223 & 0.046 & 0.114 & 0.412 & 0.203 & 0.320 \\
\hline Resting $\mathrm{PaCO}_{2}(\mathrm{mmHg})$ & -0.049 & 0.666 & 0.177 & 0.200 & -0.231 & 0.256 \\
\hline$V_{\mathrm{T}}$ at peak/SVC $(\%)$ & 0.619 & $<0.001$ & 0.485 & $<0.001$ & 0.554 & 0.003 \\
\hline Increase of peak- $V_{\mathrm{T}}$ over baseline $(\%)$ & 0.657 & $<0.001$ & 0.543 & $<0.001$ & 0.578 & 0.002 \\
\hline $\mathrm{SpO}_{2}$ at peak $(\%)$ & 0.281 & 0.012 & -0.151 & 0.276 & 0.204 & 0.318 \\
\hline
\end{tabular}

$r=$ Pearson's correlation coefficient

For abbreviations, see Tables 1 and 2 


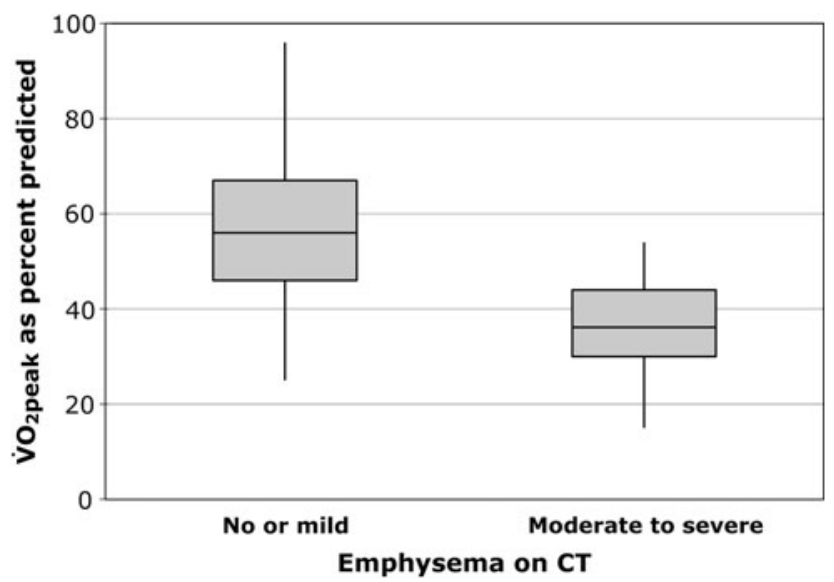

Fig. 3 Box and whisker plot of oxygen uptake at peak exercise $\left(\dot{V} \mathrm{O}_{2 \text { peak }}\right)$ in COPD patients as a function of the presence and severity of emphysema on computed tomography (CT). Line in box median. Box height interquartile range. Whiskers extreme values. $p<0.001$ by Mood's median test

peak $V_{\mathrm{T}}(r=0.682)$, resting IC $(r=0.451)$, and $\mathrm{DL}_{\mathrm{CO}}$ $(r=0.437)$. Curtailment of $V_{\mathrm{T}}$ response to exercise is ascribed primarily to dynamic hyperinflation, but no relationship is established between changes in peak $V_{\mathrm{T}}$ and severity of structural emphysema as reflected by radiologic imaging [17].

In our study, peak $V_{\mathrm{T}}$ increased by a median value of $133 \%$ over baseline in the patients with no or mild emphysema as opposed to only $75 \%$ in those with moderate to severe emphysema.

With multiple quantile regression, we find that the effects of moderate to severe emphysema on the three quartiles of $\dot{V} \mathrm{O}_{2 \text { peak }}$ are only partially explained by the degree of lung hyperinflation (IC/TLC ratio), a substantial component being imputable to the impaired lung diffusing capacity.

In normal subjects, the diffusing capacity of the lung increases during exercise mainly because of an increase in pulmonary capillary blood volume [18]. In emphysemic patients, the pulmonary capillary blood volume is diminished to a variable extent due to the loss of alveoli and alveolar capillaries [19]. Therefore, it is physiologically plausible that the impairment of lung diffusing capacity contributes substantially in limiting exercise capacity in the patients with moderate to severe emphysema.

Despite this, our findings indicate that the degree of lung hyperinflation also contributes in reducing exercise tolerance in COPD patients, as suggested by other investigators $[16,17]$.

Vassaux et al. [20] measured oxygen pulse at rest and at peak exercise in 87 patients with severe or very severe COPD, and in 46 age- and sex-matched controls. They find that patients with very severe hyperinflation at rest
$(\mathrm{IC} / \mathrm{TLC} \leq 25 \%)$ have significantly lower peak oxygen pulse than both patients with IC/TLC $>25 \%$ and controls.

The authors conclude that reduced cardiac performance, as reflected by the lower oxygen pulse, contributes to exercise limitation in severely hyperinflated COPD patients. In that study, however, the severity of emphysema was not established because there was no radiologic assessment [20].

Jörgensen et al. [21] measured intrathoracic blood volume, and left and right ventricular dimensions by magnetic resonance imaging in 13 patients with very severe emphysema and 11 healthy volunteers.

They observe that intrathoracic blood volume is significantly reduced in emphysemic patients when compared with normal subjects, and so are the end-diastolic ventricular volume index, and stroke volume index. The authors reason that the decreased biventricular preload in emphysemic patients might be due to intrathoracic hypovolemia brought about by severe lung hyperinflation [21].

In our study, peak oxygen pulse is significantly lower in the patients with moderate to severe emphysema than in those with no or mild emphysema.

The use of oxygen pulse as an index of stroke volume requires the assumption that the arterial-mixed venous oxygen difference be maximal and constant [11]. This may not be true in the patients, who desaturate during exercise [11]. Because we did not measure the arterial-mixed venous oxygen gradient, we felt it inappropriate to use peak oxygen pulse as a surrogate of cardiac performance. Nevertheless, we do not exclude the possibility that cardiac dysfunction may contribute to limit exercise tolerance in COPD patients with very severe emphysema.

In summary, we find that patients with COPD and moderate to severe emphysema feature significantly lower exercise tolerance than those with no or mild emphysema. This may be somewhat expected because the patients with overt emphysema have greater impairment of baseline lung function than the others. Yet, it seems to have little impact on the behavior of the practicing physicians. In fact, the patients reported on here were all uniformly treated by their physicians with inhaled bronchodilators and inhaled corticosteroids according to the guidelines on COPD management [1]. Nearly $70 \%$ of the emphysemic patients were on triple therapy including LABA, LAMA, and ICS (see online data supplement). In these patients, such "standard" treatment appears ineffective in improving exercise tolerance.

Our data underscore the need for the tailoring of therapeutic interventions for COPD to the predominant clinical phenotype to improve exercise tolerance and quality of life. In particular, we believe that randomized trials are warranted to establish whether pulmonary rehabilitation programs are more effective than standard pharmacological 
therapy in COPD patients with a predominant emphysema phenotype.

In the present study, volumetric CT of the thorax was used as the reference standard for diagnosing emphysema. In our experience, digital chest radiography is a valuable alternative to the $\mathrm{CT}$ scan in routine clinical practice, provided that explicit diagnostic criteria are used [5].

Recently, a neural network-based, computer-aided method was introduced that allows online recognition of emphysema on digital chest radiographs [22]. This method, validated against $\mathrm{CT}$, might be used advantageously by clinicians because it is simple and inexpensive. Its application may reduce the costs of the diagnostic procedures for emphysema, and minimize the radiation burden to the patient.

Acknowledgments The authors wish to thank Guido Nassi, Cristina Carli, and Luca Serasini for their excellent technical assistance. Permission was obtained from those who are acknowledged. This work was supported by funds from the National Research Council of Italy, and the Department of Medical and Surgical Critical Care, University of Florence, Italy.

Conflict of interest None.

\section{References}

1. Rabe KF, Hurd S, Anzueto A, Barnes PJ, Buist SA, Calverley P, Fukuchi Y, Jenkins C, Rodriguez-Roisin R, van Weel C, Zielinski J (2007) Global Initiative for Chronic Obstructive Lung Disease. Global strategy for the diagnosis, management, and prevention of chronic obstructive pulmonary disease: GOLD executive summary. Am J Respir Crit Care Med 176:532-555

2. Kitaguchi Y, Fujimoto K, Kubo K, Honda T (2006) Characteristics of COPD phenotypes classified according to the findings of HRCT. Respir Med 100:1742-1752

3. Makita H, Nasuhara Y, Nagai K, Ito Y, Hasegawa M, Betsuyaku T, Onedera Y, Hizawa N, Nashimura M, Hokkaido COPD Cohort Study (2007) Characterisation of phenotypes based on severity of emphysema in chronic obstructive pulmonary disease. Thorax 62:932-937

4. Pistolesi M, Camiciottoli G, Paoletti M, Marmai C, Lavorini F, Meoni E, Marchesi C, Giuntini C (2008) Identification of a predominant COPD phenotype in clinical practice. Respir Med 102:367-376

5. Miniati M, Monti S, Stolk J, Mirarchi G, Falaschi F, Rabinovich R, Canapini C, Roca J, Rabe KF (2008) Value of chest radiography in phenotyping chronic obstructive pulmonary disease. Eur Respir J 31:509-515

6. Ogawa E, Nakano Y, Ohara T, Muro S, Hirai T, Sato S, Sakai H, Tsukino M, Kinose D, Nishioka M, Niimi A, Chin K, Paré PD, Mishima M (2009) Body mass index in male patients with COPD: correlation with low attenuation areas on CT. Thorax $64: 20-25$
7. Jones PW, Quirk FH, Baveystock CM, Littlejohn P (1992) A selfcomplete measure of health status for chronic airflow limitation. The St. George's respiratory questionnaire. Am Rev Respir Dis 145:1321-1327

8. Miller MR, Hankinson J, Brusasco V, Burgos F, Casaburi R, Coates A, Crapo R, Enright P, van der Grinten CP, Gustafsson P, Jensen R, Johnson DC, Macintyre N, McKay R, Navajas D, Pedersen OF, Pellegrino R, Viegi G, Wanger J, ATS/ERS Task Force (2005) Standardisation of spirometry. Eur Respir J $26: 319-338$

9. Wanger J, Clausen JL, Coates A, Pedersen OF, Brusasco V, Burgos F, Casaburi R, Crapo R, Enright P, van der Grinten CP, Gustafsson P, Hankinson J, Jensen R, Johnson D, Macintyre N, McKay R, Miller MR, Navajas D, Pellegrino R, Viegi G (2005) Standardisation of the measurement of lung volumes. Eur Respir J 26:511-522

10. Macintyre N, Crapo RO, Viegi G, Johnson DC, van der Grinten CP, Brusasco V, Burgos F, Casaburi R, Coates A, Enright P, Gustafsson P, Hankinson J, Jensen R, McKay R, Miller MR, Navajas D, Pedersen OF, Pellegrino R, Wanger JI (2005) Standardisation of the single-breath determination of carbon monoxide uptake in the lung. Eur Respir J 26:720-735

11. ATS/ACCP statement on cardiopulmonary exercise test (2003) Am J Respir Crit Care Med 167:211-277

12. Wasserman K, Hansen JE, Sue DY, Stringer WW, Whipp BJ (2005) Principles of exercise testing and interpretation, 4th edn. Lippincott Williams and Wilkins, Philadelphia

13. Borg CAV (1982) Psycophysical basis of perceived exertion. Med Sci Sports 14:511-515

14. Beigelman-Aubry C, Hill C, Guibal A, Savatovsky J, Grenier PA (2005) Multi-detector row CT and postprocessing techniques in the assessment of diffuse lung disease. Radiographics 25:16391652

15. Thurlbeck WM, Dunnill MS, Hartung W, Heard BE, Heppleston AG, Ryder RC (1970) A comparison of three methods of measuring emphysema. Hum Pathol 1:215-226

16. Diaz O, Villafranca C, Ghezzo H, Borzone G, Leiva A, MilicEmili J, Lisboa C (2000) Role of inspiratory capacity on exercise tolerance in COPD patients with and without tidal expiratory flow limitation at rest. Eur Respir J 16:269-275

17. O'Donnell DE, Revill SM, Webb KA (2001) Dynamic hyperinflation and exercise intolerance in chronic obstructive pulmonary disease. Am J Respir Crit Care Med 164:770-777

18. Bates DV, Varvis CJ, Donevan RE, Christie RV (1960) Variations in the pulmonary capillary blood volume and membrane diffusion component in health and disease. J Clin Invest 39:1401-1412

19. Morrison NJ, Abboud RT, Müller NL, Miller RR, Gibson NN, Nelems B, Evans KG (1990) Pulmonary capillary blood volume in emphysema. Am Rev Respir Dis 141:53-61

20. Vassaux C, Torre-Bouscoulet L, Zeineldine S, Cortopassi F, PazDiaz H, Celli BR, Pinto-Plata VM (2008) Effects of hyperinflation on the oxygen pulse as a marker of cardiac performance in COPD. Eur Respir J 32:1275-1282

21. Jörgensen K, Müller MF, Nel J, Upton RN, Houltz E, Ricksten SE (2007) Reduced intrathoracic blood volume and left and right ventricular dimensions in patients with severe emphysema: an MRI study. Chest 131:1050-1057

22. Miniati M, Coppini G, Monti S, Bottai M, Paterni M, Ferdeghini EM (2010) Computer-aided recognition of emphysema on digital chest radiography. Eur J Radiol 